\title{
Synthesis of Circular Array Antenna for Sidelobe Level and Aperture Size Control Using Flower Pollination Algorithm
}

\author{
V. S. S. S. Chakravarthy Vedula, ${ }^{1}$ S. R. Chowdary Paladuga, ${ }^{1}$ and M. Rao Prithvi ${ }^{2}$ \\ ${ }^{1}$ Department of ECE, Raghu Institute of Technology, Visakhapatnam 531162, India \\ ${ }^{2}$ Department of ECE, Andhra University, Visakhapatnam 530003, India \\ Correspondence should be addressed to V. S. S. S. Chakravarthy Vedula; sameersree@gmail.com
}

Received 18 March 2015; Revised 18 July 2015; Accepted 30 July 2015

Academic Editor: Stefano Selleri

Copyright @ 2015 V. S. S. S. Chakravarthy Vedula et al. This is an open access article distributed under the Creative Commons Attribution License, which permits unrestricted use, distribution, and reproduction in any medium, provided the original work is properly cited.

\begin{abstract}
Sidelobe level suppression is a major problem in circular array antenna (CAA) synthesis. Many conventional numerical techniques are proposed to achieve this which are time consuming and often fail to handle multimodal problems. In this paper, a method of circular array synthesis using nature inspired flower pollination algorithm (FPA) is proposed. The synthesis technique considered here adapts one and two degrees of freedom, namely, amplitude only and amplitude spacing. The effectiveness of the FPA is studied by comparing the results with genetic algorithm $(\mathrm{GA})$ and uniform circular array antenna (UCAA) with uniform spacing. Also the effect of additional degree of freedom on the aperture size and the computational time is analyzed. A relative side lobe level (SLL) of $-25 \mathrm{~dB}$ is achieved using the algorithm under both no beam scanning $\left(0^{\circ}\right)$ and beam scanning $\left(15^{\circ}\right)$ conditions for 20 and 40 elements of CAA.
\end{abstract}

\section{Introduction}

Directivity is considered as the significant property of the radiating element [1]. The ratio of the radiation intensity in a given direction to the radiation intensity averaged over all directions [2]. Enhancing the directivity is a challenging task in antenna design. Extending the physical length of the antenna is a common practice to increase the directivity. But this technique has a direct impact on the operating (resonant) frequency which is not a desirable case as most of the antennas for wireless communications are frequency dependent [3].

Many methods are proposed to achieve good directivity without altering the physical length of the antenna. Assuming suitable geometry of reflectors is one of the techniques whereas the most reliable technique is the concept of antenna arrays. Antenna array is a set of similar antenna elements collectively operating as a single radiating element. By using antenna arrays, the physical length of the individual element in the array is unaltered but the electrical length, which is proportional to the length of the array, is extended which results in enhanced directivity. In this case, there is no change in the operating frequency as the individual element in the array is not disturbed. Antenna arrays play a significant role in modern wireless communication systems with their definite characteristics like excellent directivity and beam steering capabilities. These characteristics are favourable for applications like beam forming and null positioning. Linear, circular, planar, and cylindrical arrays are different classifications of antenna arrays with respect to the arrangement of the radiating elements in the array.

Circular arrays have become popular with the recent advancements in wireless communications. Though the design of circular arrays involves complex calculations, it has beam steering as inherent property which makes it a convenient candidate for beam forming. Every element in the circular array is characterised by three parameters, namely, excitation amplitude $(I)$, phase $(\varnothing)$, and interelement spacing $(d)$. These three are known as steering parameters in array design. They are capable enough of modifying the array radiation pattern. From synthesis point of view, they are called three degrees of freedom. Circular array design 
problem involves defining weights for one or more of these steering parameters for each element in the array, such that the resultant radiation pattern is close to the desired one.

Many numerical techniques and methods are proposed earlier, which involved pattern synthesis with nonuniform excitation amplitudes [4,5], phase adjustments [6, 7], and nonuniform spacing parameters [8]. Huge complexity is associated with such methods and always prone to stuck in the local minima. As an alternative to this, many evolutionary and heuristic approaches are proposed. The optimization techniques like genetic algorithm (GA) [9], particle swarm optimization (PSO) [10], differential evolution (DE) [11], ant colony optimization (ACO) [12], teaching learning based optimization (TLBO) [13, 14], cuckoo search (CS) [15], firefly (FF) [16], invasive weed optimization [17], and backscattering algorithms [18] have evolved in the recent years for solving antenna array optimization problems. When compared with the classical techniques, these methods have outperformed in terms of computational time and problem handling capabilities. In particular, the performance of the novel nature inspired techniques dominated the classical approach when multimodal problems are considered.

Very low SLL of $-29 \mathrm{~dB}$ is reported in [19] by adjusting the amplitude of excitation and its corresponding phase using real coded GA. Among the three parameters $(I, \varnothing$, and $d$ ) handling phase involves a huge complexity as it is not always convenient to implement fractional phase in practice. Though inclusion of phase has many advantages mathematically, it is preferred to avoid considering the practical problems. Nonuniform amplitude distribution in circular arrays has produced considerable suppression in SLL. But when the beam is scanned to certain scanning angle there is an increase in SLL. Considerable relaxation is always given to SLL when the beam is scanned to certain angle or when trying to control the BW or aperture size. Hence, there is always a need to observe the same SLL in both scanned and unscanned main beams.

As reported in [20], using GA nonuniform circular arrays with various scanning ranges could produce patterns with reduced SLL as low as $-8.1 \mathrm{~dB}$. Both amplitude and spacing are adjusted in synthesis process. Later, the same author incorporated the BW constraint along with SLL with no beam steering [21] and observed a very low SLL of $-11.8 \mathrm{~dB}$ with a 12-element asymmetric array with nonuniform excitation and nonuniform spacing. Modified invasive weed optimization (IWO) technique is employed for nonuniform circular array synthesis [22] to produce array patterns with reduced SLL of $-14.23 \mathrm{~dB}$ and improved directivity of $9.14 \mathrm{~dB}$. The results are compared to be better than classical IWO, PSO, and GA in the case of 12-element circular array in terms of SLL and directivity and also in terms of performance, evaluated from the computational time. The other simple technique to achieve better SLL in circular array is to increase number rings to form a concentric circular array [23-25].

In this paper, nature inspired flower pollination algorithm (FPA) that mimics the pollination process in flower plants is presented for circular array antenna synthesis. The algorithm is the brainchild of Yang et al. [26] and is applied for many multiobjective problems in several disciplines. In the current work, two types of controlling parameters are considered for the CAA synthesis. In the first case, amplitude only is considered and later, along with amplitude, interelement spacing is also included. In both cases, the efficiency of the flower pollination is compared with the uniformly excited and uniformly spaced circular array. The work presented proposes new algorithm for electromagnetic applications and also gives an emphasis to array antenna design with additional degree of freedom.

The entire simulation is divided into four cases. In the first two cases, amplitude only synthesis method is considered. Along with amplitude, interelement spacing is also included for synthesis in the remaining two cases. The objective of the first two cases is only to observe suppressed SLL as low as $-25 \mathrm{~dB}$ and the corresponding aperture size is same as that of UCAA, whereas the objective of the last two cases is to observe the minimised SLL with optimized aperture size less than that of UCAA. The effect of the nonuniform spacing on the aperture size with SLL constraint can be inferred from Cases 3 and 4 . The principal beam is scanned to $15^{\circ}$ angle in all even numbered cases.

The rest of the paper is organised as follows. The array design formulation is presented in Section 2 and the fitness function subject to objectives of SLL minimization is formulated in Section 3. A brief introduction to the flower pollination algorithm is presented in Section 4 and its implementation for CAA synthesis is illustrated in Section 5. Simulation results pertaining to the objective are presented in four cases in Section 6. Finally, conclusion is given in Section 7.

\section{Formulation of Array Factor}

Let us consider that the isotropic elements of the array are distributed along the circumference of the circle of radius " $r$ " as shown in Figure 1. The objective of the current problem is to find appropriate set of excitation amplitudes and interelement spacing for the elements in the array. Hence, the formulation of array factor for such an arrangement should have scope of nonuniform amplitude and nonuniform spacing distribution.

Accordingly, the array factor of the above geometry is given as [27]

$$
\operatorname{AF}(\theta)=\sum_{n=1}^{N} I_{n} \exp \left(j\left(k r \cos \left(\theta-\phi_{n}\right)+\beta_{n}\right)\right)
$$

where $n$ is element number, $N$ is number of elements in the array, $I_{n}$ is the current excitation of the $n$th element, $\beta_{n}$ is the phase excitation of the $n$th element, $d_{n}$ is the spacing factor of the $n$th element, and " $k r$ " and $\phi_{n}$ are given as

$$
\begin{aligned}
& k r=\frac{2 \pi r}{\lambda}=\sum_{i=1}^{N} d_{i}, \\
& \phi_{n}=\frac{2 \pi}{k r} \sum_{i=1}^{n} d_{i} .
\end{aligned}
$$






FIGURE 1: Nonuniform planar circular array geometry in the $x-y$ axis.

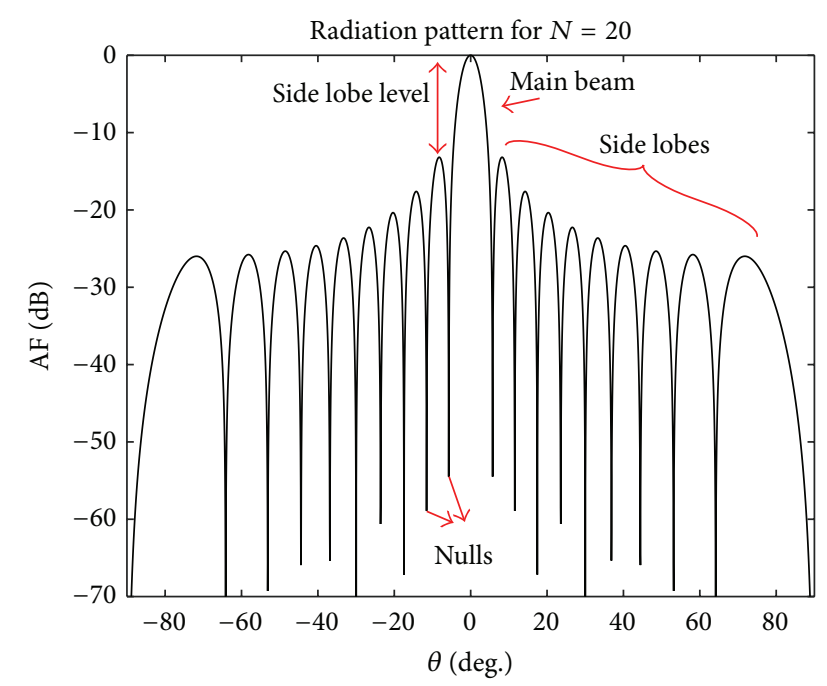

FIgURE 2: Template radiation pattern.

\section{Fitness Formulation}

The formulation of the fitness function corresponding to the objective of the work is presented in this section. Magnitude of the SLL and measured first nulls (FN) from the radiation pattern plot constitute the objective function. SLL and FN values can be extracted from the radiation pattern plot as shown in Figure 2.

The fitness function is given as

$$
f=\operatorname{SLL}_{\text {desired }}-\max \left(|\operatorname{AF}(\theta)|_{-90^{\circ} \text { to } \theta_{\mathrm{FNl}}}^{\theta_{\mathrm{FNr}} \text { to } 90^{\circ}}\right)
$$

where $\mathrm{SLL}_{\text {desired }}$ is positive value of the desired SLL. For example, in this paper, the desired SLL is $-25 \mathrm{~dB}$ and hence $\mathrm{SLL}_{\text {desired }}=25 . \theta_{\mathrm{FNl}}$ and $\theta_{\mathrm{FNr}}$ are the left and right first nulls, respectively. The $\mathrm{FN}$ is characterised by these two expressions.
The expression results in a single positive error value. Convergence is said to be achieved if this value minimises to zero.

\section{Features of Flower Pollination}

Green carpet of the nature mostly survives with the plants of flowering species. It is an acceptable fact that most of plants belong to class that survive and evolve using flowers. The very purpose of the flowers is to facilitate reproduction. Fertilization is responsible for reproduction and evolution of species. In plant species, pollination is the prerequisite of fertilization. It is the process by which pollens migrate and meet the pollen of another flower of the same plant or other plants of the same species resulting in successful fertilization.

The facilitator of pollination is known as pollinator. In biotic pollination, insects and some birds act as pollinators and transfer the pollen grains from one flower to the other. There is another form of pollination known as abiotic in which the pollen grains are transferred by wind or simple diffusion. In the nature, most of the pollination is biotic. Floral plants are reservoirs of nectar which is the source of protein for the bees. Bees approach flowers to acquire this nectar frequently and plants adapt to it such that these bees act as their pollinators resulting in genetic crossing. In this way, a mutualism builds up between the flower and the frequently visiting bees. This mutualism is called floral constancy [28], by which the bees restrict themselves to a set of floral plants and flowers depend on these bees mostly for successful pollination. This limits the memory and energy consumption of the pollinators. Pollination can be local or global. Selfpollination can be treated as local pollination in which the pollen of a flower is shared by the same flower or another flower of the same plant. Biotic cross-pollination, which takes place over long distances, is known as global pollination.

In optimization problems, pollen is considered as a solution $i$, the corresponding solution vector is given as $x_{i}$, and the solution space being the whole flower population is considered. The choice among the self-pollination and global pollination is defined by the probability switching. Fruitful pollination and successful reproduction are made possible with either of the two pollination techniques which results in the most fittest $\left(g_{*}\right)$. For updating $x_{i}$, the following mathematical representation of flower constancy is used [26]:

$$
x_{i}^{k+1}=x_{i}^{k}+L\left(x_{i}^{k}-g_{*}\right),
$$

where " $L$ " is typically referred to as step size that determines the pollination strength and is always positive and nonzero. Step size " $L$ " is characterized as "Levy Flights," [29-32] rather than a simple random number in order to mimic the movement of the biotic pollinators that are capable of sweeping long distances. The expression for $L$ is directly taken from [26] without any modification.

\section{Implementation of Flower Pollination Algorithms for Circular Arrays}

Like every population based algorithm, the starting point of FPA is population initialization. " $M$ " individuals are used 
as population. Each individual has its own solution in the $N$-dimensional solution space. $N$ also refers to the number of design variables. Hence, each solution is a set of $N$ dimensional vectors which is given as follows.

Initial population:

$$
\text { pop }=\left[x_{1}(k), x_{2}(k), \ldots, x_{M}(k)\right],
$$

where " $k$ " is the iteration number and

$$
\left[\begin{array}{c}
x_{1} \\
x_{2} \\
\cdot \\
\cdot \\
x_{M}
\end{array}\right]=\left[\begin{array}{c}
I_{1}^{1}, I_{1}^{2}, \ldots, I_{1}^{N} \\
I_{2}^{1}, I_{2}^{2}, \ldots, I_{2}^{N} \\
\vdots \\
\vdots \\
I_{M}^{1}, I_{M}^{2}, \ldots, I_{M}^{N}
\end{array}\right]
$$

where " $I$ " is current excitation coefficients.

When both current excitation and interelement spacing are used, (6) is modified in such a way that " $x$ " is vector of dimension " $2 N$." The first $N$ values are used as current excitations and the remaining $N$ are used as interelement spacing for the corresponding element:

$$
\left[\begin{array}{c}
x_{1} \\
x_{2} \\
\cdot \\
\cdot \\
x_{M}
\end{array}\right]=\left[\begin{array}{c}
I_{1}^{1}, I_{1}^{2}, \ldots, I_{1}^{N}, d_{1}^{1}, d_{1}^{2}, \ldots, d_{1}^{N} \\
I_{2}^{1}, I_{2}^{2}, \ldots, I_{2}^{N}, d_{2}^{1}, d_{2}^{2}, \ldots, d_{2}^{N} \\
\vdots \\
\vdots \\
I_{M}^{1}, I_{M}^{2}, \ldots, I_{M}^{N}, d_{M}^{1}, d_{M}^{2}, \ldots, d_{M}^{N}
\end{array}\right] .
$$

Implementation of the algorithm for array synthesis involves considering each individual that corresponds to an array of " $N$ " elements. Numerical value representing the element is the current excitation coefficient of that element. Finally, the fitness is evaluated for each individual and the best among them is supposed to be the individual with minimum fitness. This is given as [31]

$$
x^{*}(k)=\arg \min _{m=1, \ldots, M} f\left(x_{m}(k)\right) .
$$

\section{Results}

Simulation is divided into four cases as discussed earlier in order to illustrate the potency of the proposed algorithm. The weights obtained and the corresponding radiation pattern plots are given for each case. All the cases consider nonuniform amplitude distribution and Cases 3 and 4 have nonuniform spacing in addition. The objective of all the cases is to observe a very low SLL of $-25 \mathrm{~dB}$. The radiation patterns obtained using FPA are compared with those of GA and uniform CAA. Along with the minimised SLL, reduction in aperture size is also included as objective in Cases 3 and 4. It is intuitive that restricting the interelement spacing to adjust between $0.25 \lambda$ and $0.5 \lambda$ would explicitly reduce aperture size when compared with the uniformly spaced (0.5 $\lambda$ ) CAA. This technique gives an increased emphasis towards array design and decreases the stress on algorithm in achieving the objective.

For all the cases, the initial population (pop) is 20 and the switching probability $(p)$ is 0.8 . Simulation is carried out for at least 3 runs and the best is considered finally. Choice among global pollination and local pollination is determined by generating random number (rand). This is illustrated below:

$$
\text { if }(\text { rand }<p)
$$

generate a $N$-dimensional vector for step size according to Levy distribution

update via global pollination using the expression

$$
x_{i}^{k+1}=x_{i}^{k}+L\left(x_{i}^{k}-g_{*}\right)
$$

else

generate a random number $(r)$ with the range $[0,1]$

randomly choose two individuals " $l$ " and " $m$ " and update using the expression

$$
x_{i}^{k+1}=x_{i}^{k}+L\left(x_{l}^{k}-x_{m}^{k}\right)
$$

end

Case 1 (nonuniform amplitude distribution with uniform spacing and without beam steering). In this case, SLL suppression is observed using only amplitude adjustments. The weights obtained for the fitness function are directly substituted in array factor of circular array to obtain the radiation pattern. Radiation pattern pertaining to CAA of 20 and 40 isotropic elements is as shown in Figures 3(a) and 3(b), respectively. The effectiveness of the algorithm (solid line) can be analysed when compared with the radiation pattern (RP) of GA (dotted) and uniform CAA (dotted line) in these figures. The algorithm consistently produced SLL of $-25 \mathrm{~dB}$ even for a change in number of elements which is better than $-7 \mathrm{~dB}$ as in the case of UCAA.

Case 2 (nonuniform amplitude distribution with uniform spacing and with main beam steering). In this case, the main beam is scanned to $15^{\circ}$. The objective is to observe a SLL of $-25 \mathrm{~dB}$ which remains the same even though the main beam is scanned. The same is evident in the plots Figures 4(a) and 4(b). It is evident from the plots that the algorithm is efficient enough to maintain the same SLL for both scanned and unscanned main beam conditions.

Case 3 (nonuniform amplitude distribution with nonuniform spacing and without beam steering). Nonuniform spacing along with nonuniform amplitude distribution is considered in this case. Figures 5(a) and 5(b) represent the RP of uniform and nonuniform CAA overlapped for $N=20$ and 40, respectively. 


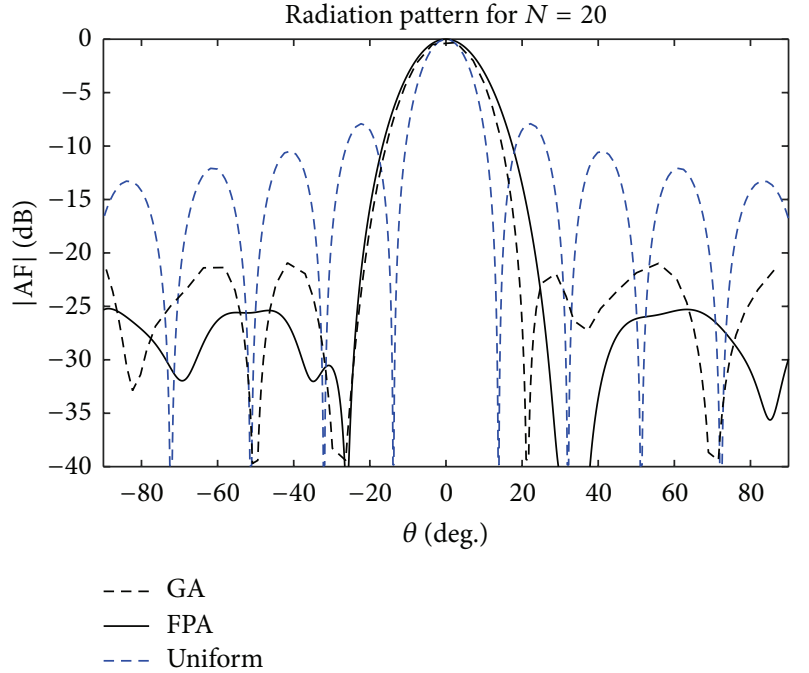

(a)

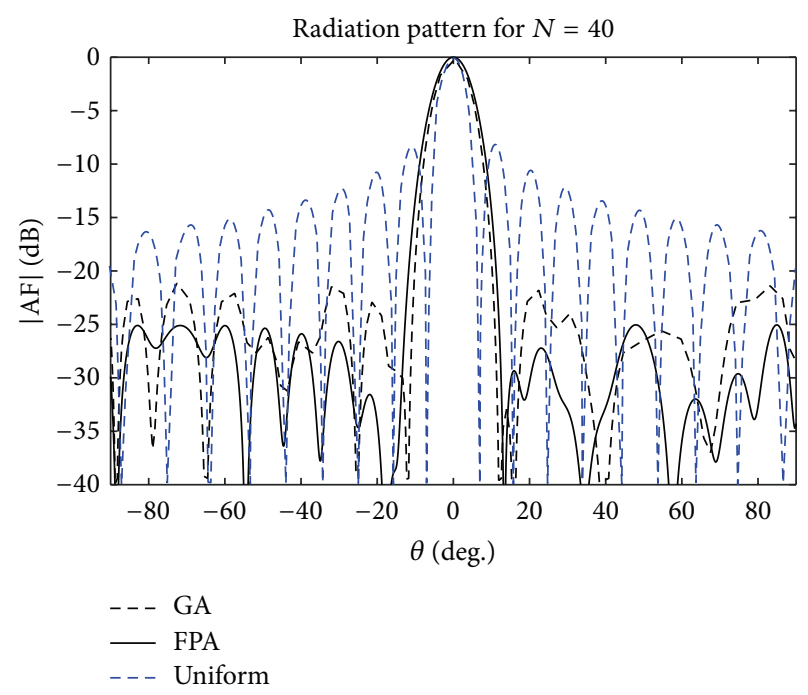

(b)

FIGURE 3: Comparison of the radiation patterns of CAA using weights obtained by FPA and uniform distribution for (a) $N=20$ and (b) $N=40$.

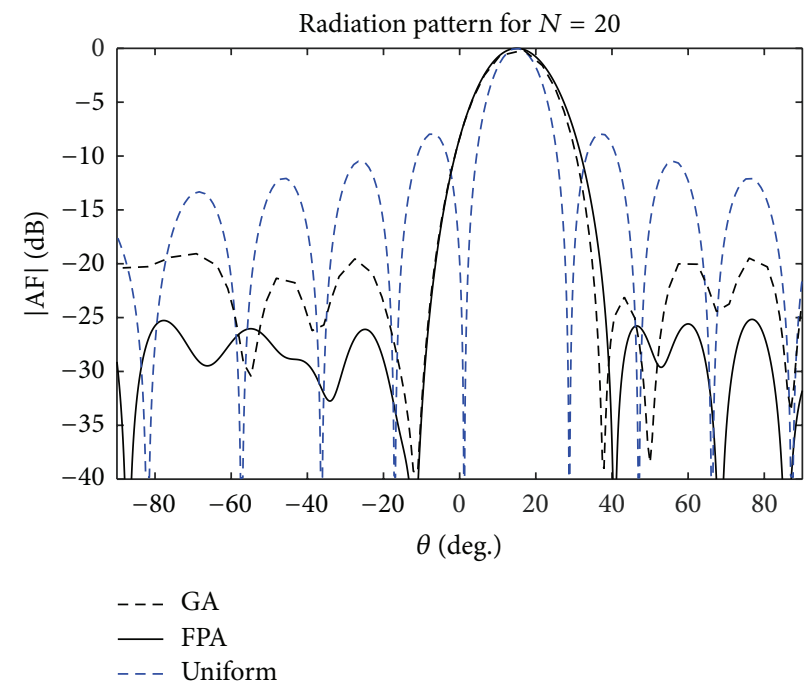

(a)

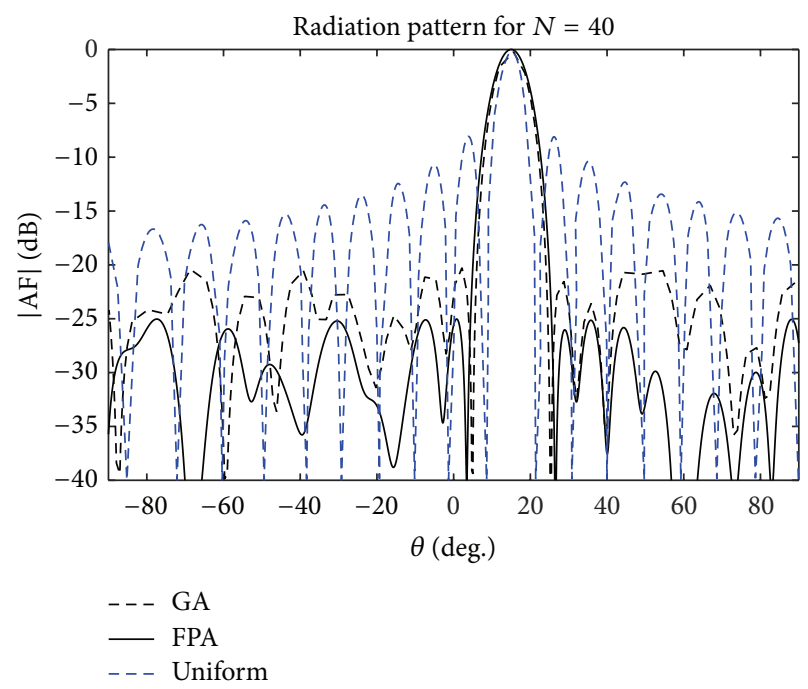

(b)

FIGURE 4: Comparison of the radiation patterns of CAA with main beam scanned to $15^{\circ}$ using weights obtained by FPA and uniform distribution for (a) $N=20$ and (b) $N=40$.

Case 4 (nonuniform amplitude distribution with nonuniform spacing and with beam steering). The amplitude and space distribution are similar to those of Case 3, whereas the main beam is scanned to $15^{\circ}$. The algorithm could successfully produce SLL of $-25 \mathrm{~dB}$ which is evident from Figures 6(a) and 6(b) for $N=20$ and 40, respectively.

Figures 5 and 6 are similar to Figures 3 and 4, respectively, in terms of objective, but the technique of array synthesis is different. The amplitude only technique is used to produce the desired radiation patterns in Figures 3 and 4. Similarly, amplitude-space technique is employed for Figures 5 and 6. The amplitude distribution for 20 and 40 element circular array in Cases 1 and 2 is given in Table 1 . The first two rows refer to the distribution when the main beam is at $0^{\circ}$ and the remaining two rows refer to the distribution when the beam is scanned to $15^{\circ}$.

Similarly the amplitude and the interelement space distribution are given in Table 2 for Cases 3 and 4. Certainly, inclusion of interelement spacing along with amplitude distribution has an advantage with the computational time for synthesis. In order to utilize this advantage completely, the typical range of the space function should be $0-2 \lambda$ [20]. This procedure would directly enhance the aperture size while reducing the computational time. To control the aperture size, the objective function should hold another parameter 


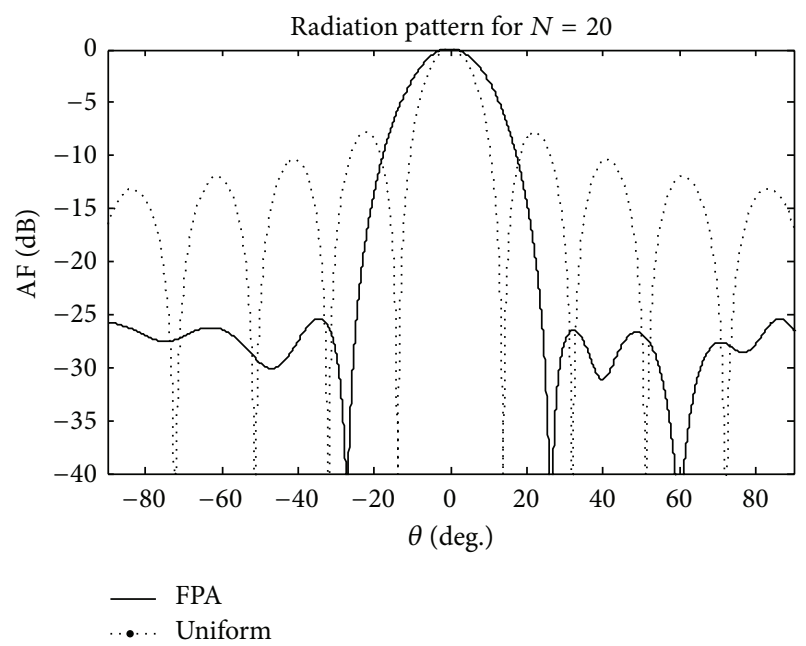

(a)

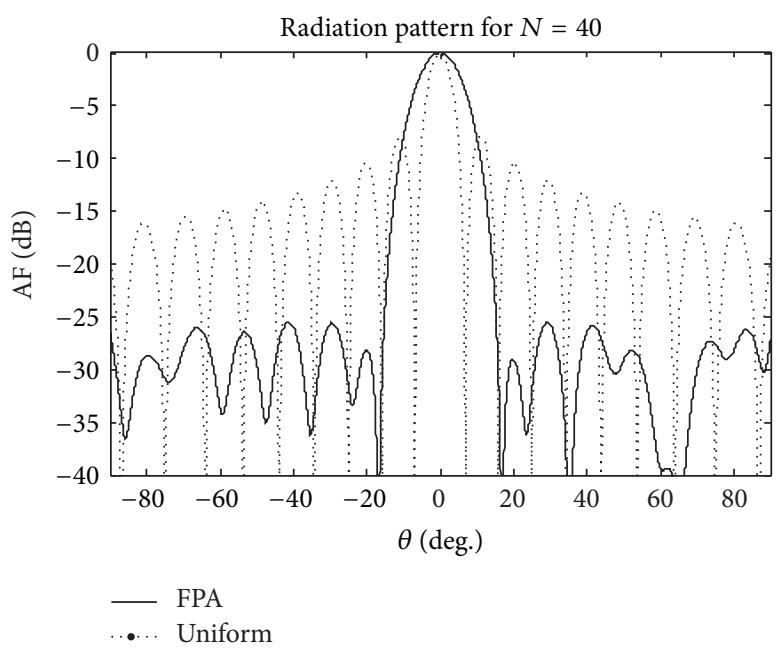

(b)

FIGURE 5: Comparison of the radiation patterns of CAA using weights obtained by FPA and uniform distribution for (a) $N=20$ and (b) $N=40$.

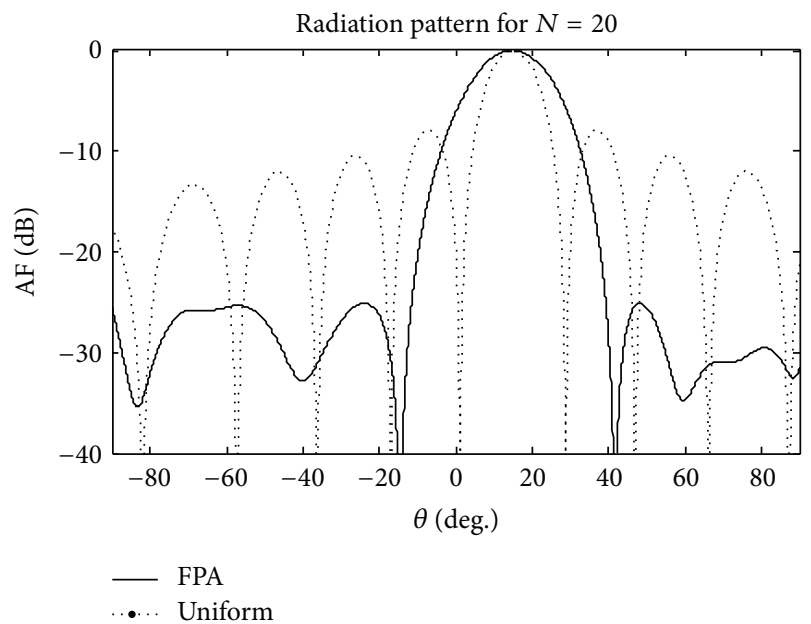

(a)

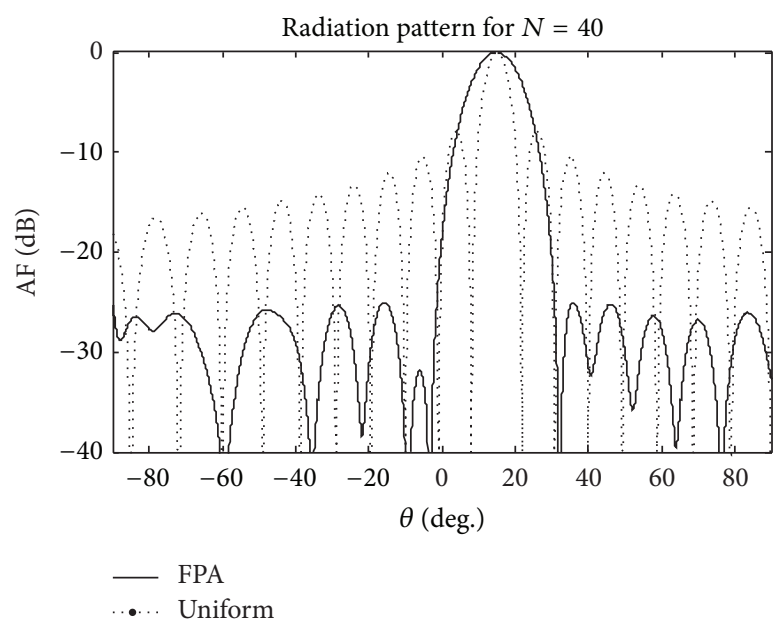

(b)

FIGURE 6: Comparison radiation patterns of CAA with main beam scanned to $15^{\circ}$ using weights obtained by FPA and uniform distribution for (a) $N=20$ and (b) $N=40$.

that controls the aperture size. In this work, an alternative method is suggested by restricting the interelement space to the range of $0.2 \lambda-0.5 \lambda$. Without much stress on the algorithm, the resultant space function definitely has aperture size less than that of uniformly spaced CAA. This comparison is consolidated in Table 3.

The fourth column refers to the aperture size when amplitude only technique is employed (refer to Cases 1 and 2) for the corresponding number of elements when the beam is at $0^{\circ}$ and $15^{\circ}$, respectively. Similarly, the aperture size can be read from the fifth column for Cases 3 and 4 for the corresponding number of elements in the row. It is clear that the values mentioned in the fifth column are less than the corresponding values in fourth column. Which means that there is a decrease in aperture size.

\section{Conclusion}

FPA is successfully implemented for circular array synthesis and compared with GA which is validated for many array design applications. A relatively very low SLL is observed with FPA when compared with GA and uniform circular array. Also it can be inferred from Cases 3 and 4 that there is direct impact on the aperture size control with the inclusion of space function. This approach avoids complexity in the objective function and simplifies the role of the algorithm. Though the implementation of GA is not performed for Cases 3 and 4, the emphasis in these cases is to elevate the role of the objective function rather than concentrating on the efficiency of the adopted algorithm. However, it can be considered that the inclusion of such strategy in the objective function would 
TABle 1: Amplitude distribution for Cases 1 and 2.

\begin{tabular}{|c|c|c|c|}
\hline $\begin{array}{l}\text { Main beam } \\
\text { direction }\end{array}$ & Number of elements & Algorithm & Amplitudes $=\left[I_{1}, I_{2}, \ldots, I_{n}\right]$ \\
\hline \multirow{4}{*}{$0^{\circ}$} & \multirow{2}{*}{20} & GA & $\begin{array}{l}0.9819,0.43433,0.38883,0.08801,0.049708,0.13402,0.23325,0.34977,0.13993,0.63811, \\
0.89366,0.32359,0.17678,0.15957,0.0296,0.15514,0.25948,0.20366,0.20818,0.79876\end{array}$ \\
\hline & & FPA & $\begin{array}{l}0.9936,0.6571,0.3843,0.1512,0.0835,0.3995,0.0039,0.0930,0.2074,0.5338,0.8838 \\
0.4216,0.2718,0.0049,0.1487,0.0008,0.2199,0.0010,0.3326,0.4126\end{array}$ \\
\hline & \multirow{2}{*}{40} & GA & $\begin{array}{l}0.99322,0.85177,0.97018,0.64356,0.74288,0.096025,0.060732,0.47024,0.035304, \\
0.17102,0.041402,0.10706,0.26983,0.22304,0.11094,0.2566,0.11416,0.83393,0.55396, \\
0.49157,0.95757,0.71068,0.78998,0.2975,0.27882,0.30547,0.14734,0.095738, \\
0.083199,0.2460,0.045152,0.0039346,0.13798,0.41212,0.021184,0.20666,0.45209, \\
0.65734,0.95256,0.84257\end{array}$ \\
\hline & & FPA & $\begin{array}{l}0.9861,0.9887,0.8966,0.3365,0.6388,0.0924,0.1034,0.2169,0.0333,0.0508,0.0128, \\
0.1327,0.0135,0.0916,0.2144,0.2084,0.5182,0.6454,0.9887,0.7422,0.9030,0.8925 \\
0.8632,0.4566,0.3390,0.2704,0.1085,0.0066,0.1799,0.0329,0.0106,0.0500,0.2899 \\
0.2200,0.0625,0.1681,0.3882,0.8378,0.9606,0.9535\end{array}$ \\
\hline \multirow{4}{*}{$15^{\circ}$} & \multirow{2}{*}{20} & GA & $\begin{array}{l}0.66762,0.83441,0.20899,0.31369,0.039093,0.11261,0.042027,0.19448,0.053112 \\
0.54955,0.78473,0.97314,0.85825,0.26283,0.051948,0.29997,0.23677,0.078657 \\
0.17409,0.37731\end{array}$ \\
\hline & & FPA & $\begin{array}{l}0.4900,0.9456,0.4478,0.1899,0.2253,0.23242,0.0447,0.0012,0.0114,0.4220,0.4843 \\
0.9290,0.5457,0.2017,0.1795,0.15560 .1597,0,0.0203,0.3511\end{array}$ \\
\hline & \multirow[b]{2}{*}{40} & GA & $\begin{array}{l}0.70345,0.75233,0.73214,0.90614,0.96208,0.53025,0.67645,0.082539,0.31248, \\
0.27426,0.15,0.59956,0.11915,0.13204,0.23781,0.52463,0.019683,0.17863,0.39147, \\
0.89913,0.64331,0.96361,0.49537,0.63018,0.89284,0.89971,0.030009,0.33951,0.17512 \text {, } \\
0.01014,0.30863,0.0093453,0.43728,0.060419,0.20309,0.023254,0.1424,0.30747, \\
0.53286,0.89469\end{array}$ \\
\hline & & FPA & $\begin{array}{l}0.6685,0.8337,0.7027,0.6188,0.8419,0.6388,0.3948,0.1016,0.0535,0.2223,0.1155 \\
0.4134,0.0000,0.0394,0.1042,0.2835,0.3935,0.0448,0.5967,0.9898,0.8380,0.8192 \\
0.7498,0.9279,0.9724,0.7594,0.1221,0.1514,0.0194,0.3006,0.1054,0.2008,0.0411 \\
0.1377,0.1772,0.4936,0.0459,0.0179,0.5315,0.9973\end{array}$ \\
\hline
\end{tabular}

TABLE 2: Amplitude and interelement space distribution for Cases 3 and 4.

\begin{tabular}{|c|c|c|c|}
\hline $\begin{array}{l}\text { Number of } \\
\text { elements }\end{array}$ & Main beam direction & Parameter & Distribution \\
\hline \multirow[b]{2}{*}{20} & \multirow{4}{*}{$0^{\circ}$} & Amps & $\begin{array}{l}0.8619,0.3195,0.0214,0.0121,0.6407,0.4229,0.0000,0.3251,0.9834,0.7771,0.9997 \text {, } \\
0.2876,0.3977,0.1240,0.4019,0.2116,0.6079,0.0596,0.9262,0.9676\end{array}$ \\
\hline & & Spacing & $\begin{array}{l}0.3369,0.4850,0.4006,0.2647,0.4963,0.4973,0.4986,0.4011,0.4996,0.3089,0.2676 \text {, } \\
0.4262,0.4700,0.4749,0.5000,0.4757,0.4911,0.4049,0.4894,0.2937\end{array}$ \\
\hline \multirow[b]{2}{*}{40} & & Amps & $\begin{array}{l}0.8811,0.8008,0.3251,0.8078,0.1487,0.0170,0.1933,0.0573,0.0085,0.3539,0.0257 \\
0.1550,0.0205,0.5005,0.3540,0.5473,0.6053,0.8268,0.7866,0.8881,0.9332,1.0000 \\
0.7673,0.6267,0.4929,0.3048,0.4683,0.0118,0.0056,0.1860,0.2041,0.0035,0.0953 \\
0.5103,0.1352,0.6457,0.5603,0.6074,0.8803,1.0000\end{array}$ \\
\hline & & Spacing & $\begin{array}{l}0.3739,0.3383,0.3003,0.4241,0.3063,0.4931,0.3607,0.4934,0.3616,0.4548,0.4625, \\
0.4656,0.4266,0.4505,0.3663,0.3698,0.3172,0.2681,0.2550,0.2802,0.4041,0.2701, \\
0.3616,0.3458,0.3372,0.4236,0.4932,0.4621,0.4771,0.3851,0.3281,0.4958,0.4397, \\
0.4842,0.4000,0.2864,0.3974,0.2546,0.3089,0.4039\end{array}$ \\
\hline \multirow{2}{*}{20} & \multirow{4}{*}{$15^{\circ}$} & Amps & $\begin{array}{l}0.6083,0.6349,0.2588,0.2339,0.0000,0.2307,0.41440 .3074,0.1940,0.8014,0.9869 \\
0.4356,0.2545,0.0476,0.0190,0.5114,0.2839,0.0987,0.1308,0.9148\end{array}$ \\
\hline & & Spacing & $\begin{array}{l}0.3369,0.2087,0.3419,0.4824,0.3906,0.4897,0.4135,0.5000,0.5000,0.4530,0.3777, \\
0.2665,0.4462,0.4343,0.5000,0.4038,0.4772,0.4571,0.2482,0.4870\end{array}$ \\
\hline \multirow[b]{2}{*}{40} & & Amps & $\begin{array}{l}0.9871,0.8319,0.9915,0.5635,0.9477,0.2038,0.6511,0.7517,0.2521,0.2027,0.0191, \\
0.0060,0.0341,0.1774,0.3761,0.0211,0.9749,0.0711,0.9982,0.7881,0.9952,0.8975, \\
0.8434,0.8898,0.6869,0.2138,0.0206,0.2775,0.2438,0.4098,0.0027,0.0016,0.0069 \text {, } \\
0.4387,0.3516,0.5479,0.4311,0.7191,0.7725,0.8217\end{array}$ \\
\hline & & Spacing & $\begin{array}{l}0.2483,0.2350,0.2984,0.2283,0.2043,0.2506,0.3278,0.4203,0.5000,0.4956,0.4921 \text {, } \\
0.4816,0.4789,0.4078,0.4466,0.2874,0.4875,0.2810,0.3805,0.3719,0.2146,0.3583, \\
0.2726,0.2919,0.4493,0.3958,0.2434,0.2372,0.2133,0.3255,0.4385,0.3550,0.4479, \\
0.4650,0.4993,0.4138,0.4156,0.2854,0.3621,0.2202\end{array}$ \\
\hline
\end{tabular}


TABle 3: Aperture size comparison.

\begin{tabular}{|c|c|c|c|c|}
\hline \multirow{2}{*}{ S. number } & \multirow{2}{*}{ Number of elements } & \multirow{2}{*}{ Direction of the principal beam } & \multicolumn{2}{|c|}{ Aperture size (in $\lambda)$} \\
\hline & & & Using amplitude only technique & Using amplitude-space technique \\
\hline 1 & 20 & \multirow{2}{*}{$0^{\circ}$} & 9.5 & 8.4833 \\
\hline 2 & 40 & & 19.5 & 15.3291 \\
\hline 3 & 20 & \multirow{2}{*}{$15^{\circ}$} & 9.5 & 9.43 \\
\hline 4 & 40 & & 19.5 & 15.3291 \\
\hline
\end{tabular}

yield the same results with any algorithm subject. Although this approach is demonstrated for circular array synthesis, it can also be applied to arbitrary arrays.

\section{Conflict of Interests}

The authors declare that there is no conflict of interests regarding the publication of this paper.

\section{References}

[1] G. Marconi, "On methods whereby the radiation of electric waves may be mainly confined to certain directions, and whereby the receptivity of a receiver may be restricted to electric waves emanating from certain directions," Proceedings of the Royal Society A: Mathematical, Physical and Engineering Sciences, vol. 77, no. 518, pp. 413-421, 1906.

[2] K. D. Cheng, "Optimization techniques for antenna arrays," Proceedings of the IEEE, vol. 59, no. 12, pp. 1664-1674, 1971.

[3] P. S. Chowdary, A. M. Prasad, P. M. Rao, and J. Anguera, "Design and performance study of sierpinski fractal based patch antennas for multiband and miniaturization characteristics," Wireless Personal Communications, vol. 83, no. 3, pp. 1713-1730, 2015.

[4] N. Goto and Y. Tsunoda, "Sidelobe reduction of circular arrays with a constant excitation amplitude," IEEE Transactions on Antennas and Propagation, vol. 25, no. 6, pp. 896-898, 1977.

[5] R. Vescovo, "Constrained and unconstrained synthesis of array factor for circular arrays," IEEE Transactions on Antennas and Propagation, vol. 43, no. 12, pp. 1405-1410, 1995.

[6] F. Watanabe, N. Goto, A. Nagayama, and G. Yoshida, "A pattern synthesis of circular arrays by phase adjustment," IEEE Transactions on Antennas and Propagation, vol. 28, no. 6, pp. 857-863, 1980.

[7] R. Vescovo, "Reconfigurability and beam scanning with phaseonly control for antenna arrays," IEEE Transactions on Antennas and Propagation, vol. 56, no. 6, pp. 1555-1565, 2008.

[8] A. L. Maffett, "Array factors with nonuniform spacing parameter," IRE Transactions on Antennas and Propagation, vol. 10, no. 2, pp. 131-136, 1962.

[9] D. E. Goldberg, Genetic Algorithms in Search, Optimization, and Machine Learning, vol. 412, Addison-Wesley, Reading, Mass, USA, 1989.

[10] J. Kennedy and R. Eberhart, "Particle swarm optimization," in Proceedings of the IEEE International Conference on Neural Networks, pp. 1942-1948, December 1942.

[11] R. Storn and K. Price, "Differential evolution-a simple and efficient heuristic for global optimization over continuous spaces," Journal of Global Optimization, vol. 11, no. 4, pp. 341359, 1997.
[12] D. Karaboga, K. Guney, and A. Akdagli, "Antenna array pattern nulling by controlling both amplitude and phase using modified touring ant colony optimization algorithm," International Journal of Electronics, vol. 91, no. 4, pp. 241-251, 2004.

[13] R. V. Rao, V. J. Savsani, and D. P. Vakharia, “Teaching-learningbased optimization: a novel method for constrained mechanical design optimization problems," Computer Aided Design, vol. 43, no. 3, pp. 303-315, 2011.

[14] V. V. S. S. S. Chakravarthy, K. Naveen Babu, S. Suresh, P. Chaya Devi, and P. Mallikarjuna Rao, "Linear array optimization using teaching learning based optimization," Advances in Intelligent Systems and Computing, vol. 338, pp. 183-187, 2015.

[15] X.-S. Yang and S. Deb, "Cuckoo search: recent advances and applications," Neural Computing and Applications, vol. 24, no. 1, pp. 169-174, 2014.

[16] X.-S. Yang, "Firefly algorithms for multimodal optimization," in Stochastic Algorithms: Foundations and Applications: 5th International Symposium, SAGA 2009, Sapporo, Japan, October 26-28, 2009. Proceedings, vol. 5792 of Lecture Notes in Computer Science, pp. 169-178, Springer, Berlin, Germany, 2009.

[17] H. Wu, C. Liu, and X. Xie, “Thinning of concentric circular antenna arrays using improved binary invasive weed optimization algorithm," Mathematical Problems in Engineering, vol. 2015, Article ID 365280, 8 pages, 2015.

[18] K. Guney, A. Durmus, and S. Basbug, "Backtracking search optimization algorithm for synthesis of concentric circular antenna arrays," International Journal of Antennas and Propagation, vol. 2014, Article ID 250841, 11 pages, 2014.

[19] K.-K. Yan and Y. Lu, "Sidelobe reduction in array-pattern synthesis using genetic algorithm," IEEE Transactions on Antennas and Propagation, vol. 45, no. 7, pp. 1117-1122, 1997.

[20] M. A. Panduro, A. L. Méndez, G. Romero, and R. F. Domínguez, "Design of non-uniform circular phased arrays using genetic algorithms to reduce the maximum side lobe during scanning," in Proceedings of the 63rd IEEE Vehicular Technology Conference (VTC '06), vol. 6, pp. 2696-2700, Melbourne, Australia, May 2006.

[21] M. A. Panduro, A. L. Mendez, R. Dominguez, and G. Romero, "Design of non-uniform circular antenna arrays for side lobe reduction using the method of genetic algorithms," AEUInternational Journal of Electronics and Communications, vol. 60, no. 10, pp. 713-717, 2006.

[22] G. G. Roy, S. Das, P. Chakraborty, and P. N. Suganthan, "Design of non-uniform circular antenna arrays using a modified invasive weed optimization algorithm," IEEE Transactions on Antennas and Propagation, vol. 59, no. 1, pp. 110-118, 2011.

[23] D. Mandal, S. P. Ghoshal, and A. K. Bhattacharjee, "Application of evolutionary optimization techniques for finding the optimal set of concentric circular antenna array," Expert Systems with Applications, vol. 38, no. 4, pp. 2942-2950, 2011. 
[24] A. Reyna, M. A. Panduro, D. H. Covarrubias, and A. Mendez, "Design of steerable concentric rings array for low side lobe level," Scientia Iranica, vol. 19, no. 3, pp. 727-732, 2012.

[25] D. Durbadal Mandal, Md. A. Iqbal Ansari, R. Kar, and S. P. Ghoshal, "Non-uniform concentric circular antenna array design using IPSO technique for side lobe reduction," Procedia Technology, vol. 6, pp. 856-863, 2012.

[26] X.-S. Yang, M. Karamanoglu, and X. He, "Flower pollination algorithm: a novel approach for multiobjective optimization," Engineering Optimization, vol. 46, no. 9, pp. 1222-1237, 2014.

[27] C. A. Balanis, Antenna Theory: Analysis and Design, John Wiley \& Sons, Singapore, 2nd edition, 2003.

[28] M. Amaya-Márquez, "Floral constancy in bees: a revision of theories and a comparison with other pollinators," Revista Colombiana de Entomología, vol. 35, no. 2, 2009.

[29] I. Pavlyukevich, "Lévy flights, non-local search and simulated annealing," Journal of Computational Physics, vol. 226, no. 2, pp. 1830-1844, 2007.

[30] X.-S. Yang and S. Deb, "Cuckoo search via Lévy flights," in Proceedings of the World Congress on Nature \& Biologically Inspired Computing (NaBIC '09), pp. 210-214, Coimbatore, India, December 2009.

[31] S. Łukasik and P. A. Kowalski, "Study of flower pollination algorithm for continuous optimization," Advances in Intelligent Systems and Computing, vol. 322, pp. 451-459, 2015.

[32] V. V. S. S. S. Chakravarthy and P. M. Rao, "On the convergence characteristics of flower pollination algorithm for circular array synthesis," in Proceedings of the 2nd International Conference on Electronics and Communication Systems (ICECS '15), pp. 485489, IEEE, Feburary 2015. 

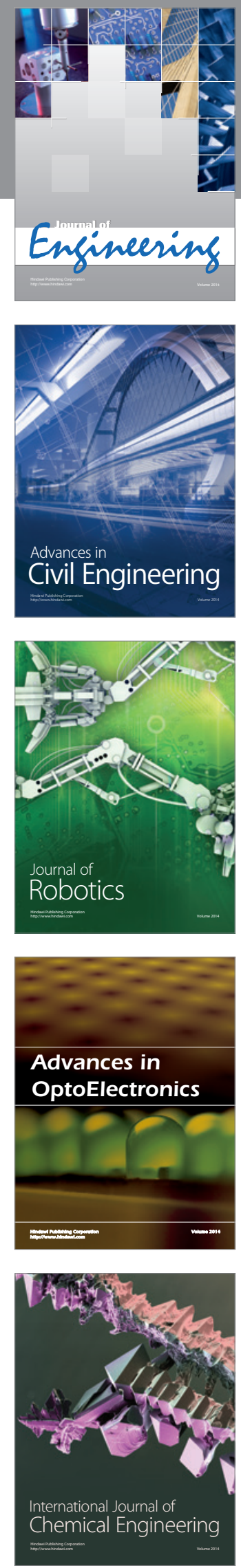

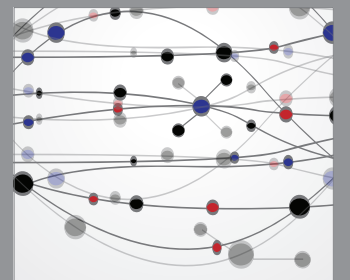

The Scientific World Journal
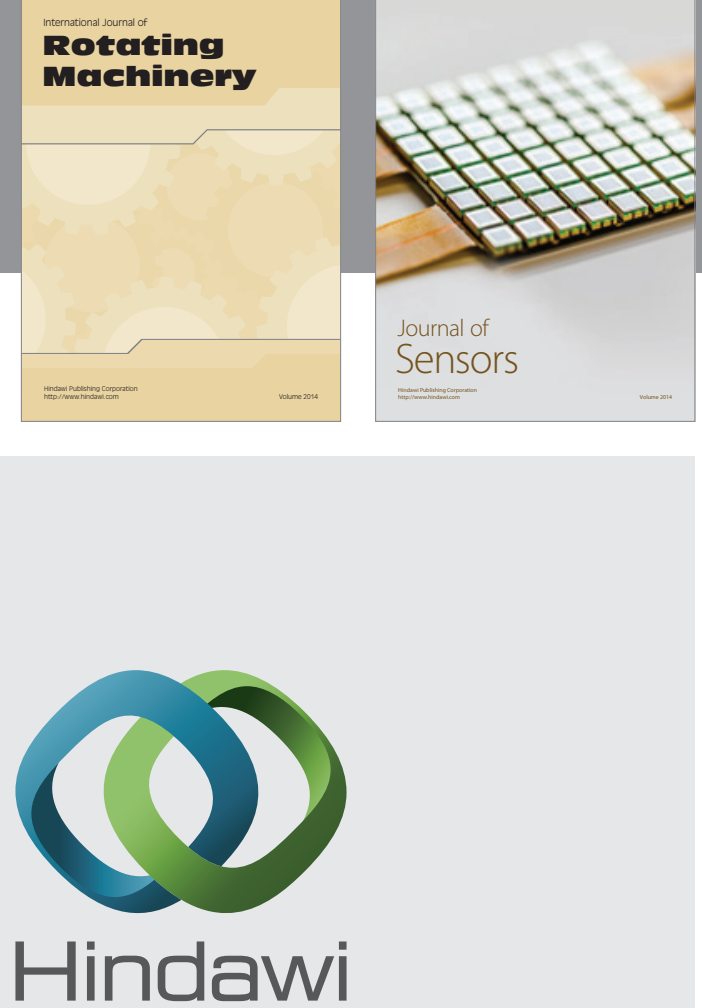

Submit your manuscripts at http://www.hindawi.com
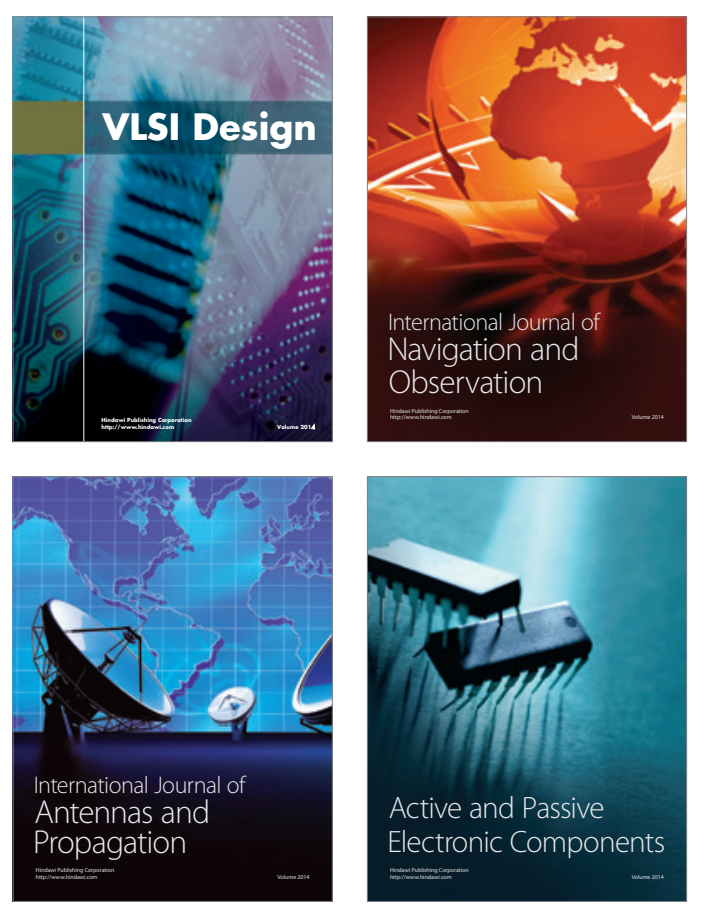
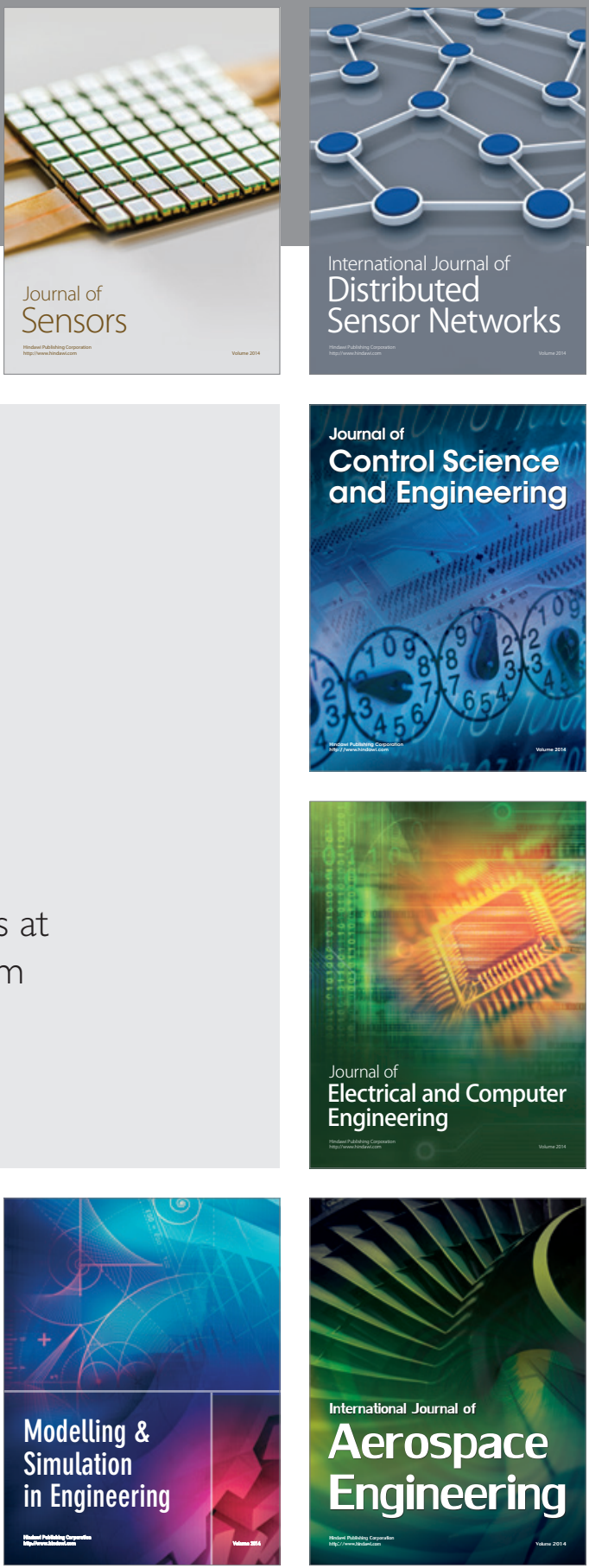

Journal of

Control Science

and Engineering
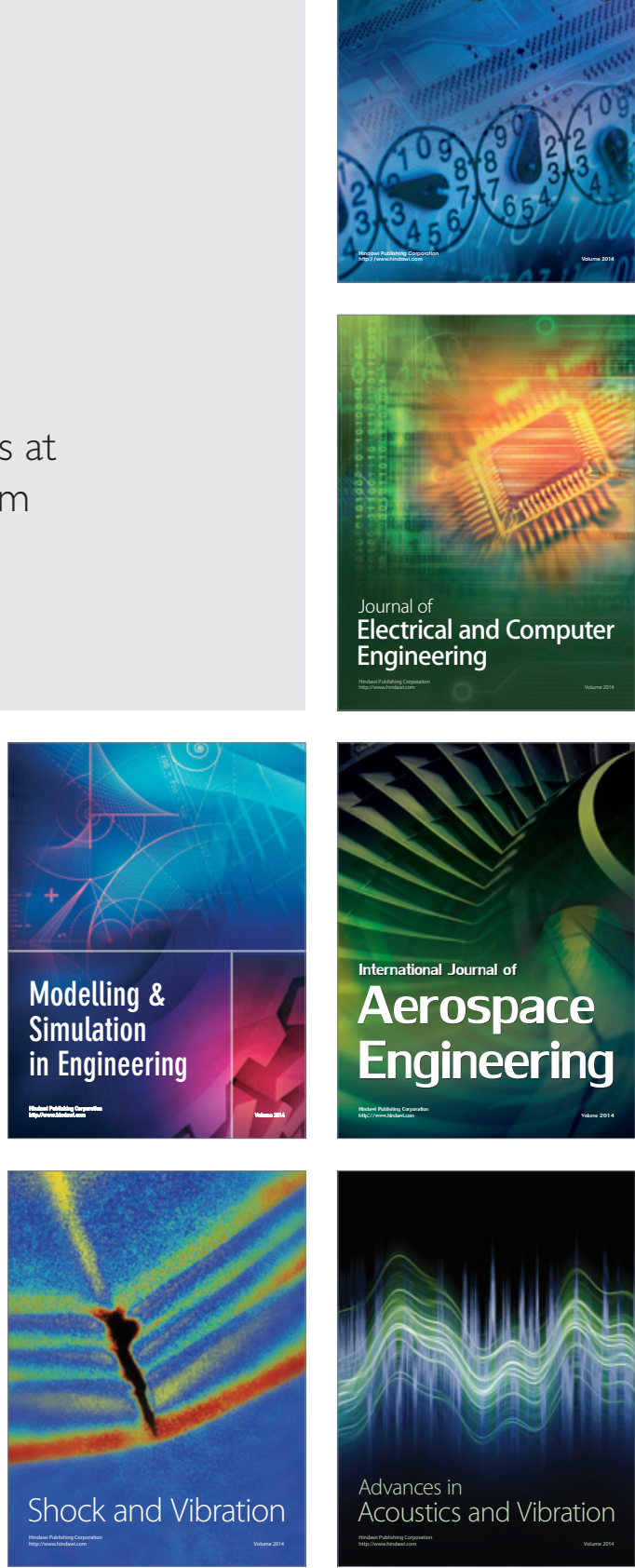